\title{
What can the detection of a single pair of circles-in-the-sky tell us about the geometry and topology of the Universe?
}

\author{
B. Mota, ${ }^{1}$ M.J. Rebouças, ${ }^{2}$ and R. Tavakol ${ }^{3}$ \\ ${ }^{1}$ Universidade Federal do Rio de Janeiro, NACO - CCS - Av. Brigadeiro Trompowski $s / n$ \\ 21941-590 Rio de Janeiro - RJ, Brazil \\ ${ }^{2}$ Centro Brasileiro de Pesquisas Físicas, Rua Dr. Xavier Sigaud 150 \\ 22290-180 Rio de Janeiro - RJ, Brazil \\ ${ }^{3}$ Astronomy Unit, School of Mathematical Sciences, Queen Mary University of London \\ Mile End Road, London, E1 4NS, UK
}

(Dated: December 6, 2018)

\begin{abstract}
In a Universe with a detectable nontrivial spatial topology the last scattering surface contains pairs of matching circles with the same distribution of temperature fluctuations - the so-called circles-in-the-sky. Searches undertaken for nearly antipodal pairs of such circles in cosmic microwave background maps have so far been unsuccessful. Previously we had shown that the negative outcome of such searches, if confirmed, should in principle be sufficient to exclude a detectable non-trivial spatial topology for most observers in very nearly flat $\left(0<\left|\Omega_{\mathrm{tot}}-1\right| \lesssim 10^{-5}\right)$ (curved) universes. More recently, however, we have shown that this picture is fundamentally changed if the universe turns out to be exactly flat. In this case there are many potential pairs of circles with large deviations from antipodicity that have not yet been probed by existing searches. Here we study under what conditions the detection of a single pair of circles-in-the-sky can be used to uniquely specify the topology and the geometry of the spatial section of the Universe. We show that from the detection of a single pair of matching circles one can infer whether the spatial geometry is flat or not, and if so we show how to determine the topology (apart from one case) of the Universe using this information. An important additional outcome of our results is that the dimensionality of the circles-in-the-sky parameter space that needs to be spanned in searches for matching pairs of circles is reduced from six to five degrees of freedom, with a significant reduction in the necessary computational time.
\end{abstract}

PACS numbers: 98.80.-k, 98.80.Es, 98.80.Jk

\section{INTRODUCTION}

The determination of the shape or topology of the Universe constitutes one of the fundamental open questions in $\operatorname{cosmology}^{1}$ (see, e.g., the reviews [1]). An important observable signature of a universe with a detectable nontrivial spatial topology is the presence in the cosmic microwave background (CMB) sky of pairs of matching circles — the so-called circles-in-the-sky — with identical distributions (up to a phase) of temperature fluctuations [2]. These pairs of circles can in turn be specified in terms of their associated parameters, as for example their radii, relative phases, and deviations from antipodicity. $^{2}$ The topology of a compact 3-manifold can be uniquely specified in terms of its holonomy group. Furthermore, each distinct pair of circles can be identified with a distinct element of this group.

Given the recent accumulation of high resolution observations that indicates that the Universe is flat or nearly flat, a great deal of effort has gone into studying the observable signatures of cosmic topology in flat and nearly

\footnotetext{
${ }^{1}$ In line with the usage in the literature, by topology of the Universe we mean the topology of its spatial sections.

2 This deviation refers to pair of circles whose centers are antipodal points on the CMB sphere, which are known as back-to-back or antipodal circles-in-the-sky.
}

flat universes (see, for example, Refs. [3]). An important outcome of our previous works has been that for detectable pairs of circles in a very nearly flat compact universe, the deviations from antipodicity will be small for most observers [4, 5]. This result, together with the negative result of recent searches for antipodal and nearly antipodal circles [6, 7], had been taken to be sufficient to exclude a detectable nontrivial topology for most observers [5]. More recently, we considered the case of an exactly flat universe. By making a detailed study of all the compact orientable flat manifolds [8], we found that the deviation from antipodicity in some compact orientable flat universes can be larger than $10^{\circ}$, i.e. outside the parameter ranges covered in the searches of Refs. [6, 7]. This result has the important consequence that if the Universe is in fact flat then the searches undertaken so far, which have confined themselves to small deviations from antipodicity, would not be sufficient to rule out the possibility of a nontrivial cosmic topology. ${ }^{3}$

\footnotetext{
3 This important difference comes ultimately from the very fact that flat, spherical and hyperbolic classes of manifolds are topologically disjoint in that their discrete topological invariants are different, and do not transform smoothly between the classes. In other words, the nonexistence of a smooth limit results comes from the fact that the classes of multiply-connected manifolds associated with positive, negative and zero spatial curvatures are not only topologically inequivalent, but also very dissimilar
} 
This possibility raises in turn the very interesting question of the extent to which the geometry and topology of the spatial sections of the Universe can be determined, if a single pair of circles is detected in any future search of $\mathrm{CMB}$ data. Here we make a systematic study of this question and show that, interestingly, from the detection of a single pair of matching circles-in-the-sky one can infer whether the spatial geometry is flat, and if so what is the specific compact orientable flat topology of the Universe (apart from one case).

The structure of the paper is as follows. In Sec. II we derive the relations between the circles-in-the sky and the holonomy parameters. In Sec. III we discuss the extent to which the geometry and topology of a universe can be specified, given the detection of a single pair of circles and its related parameters. In Sec. IV we present our final remarks and conclusions.

\section{RELATING THE CIRCLES-IN-THE-SKY AND HOLONOMY PARAMETERS}

Let us begin by recalling that homogeneous and isotropic spatial sections $M$ of flat universes are often assumed to be the simply connected euclidian 3-manifold $\mathbb{R}^{3}$. However, they can also be multiply connected quotient 3-manifolds (which we assume to be compact and orientable) of the form $M=\mathbb{R}^{3} / \Gamma$, where $\mathbb{R}^{3}$ is the covering space, and $\Gamma$ is a discrete and fixed point-free group of isometries of $\mathbb{R}^{3}$ called the holonomy group [10]. Throughout this paper a generic element of the group $\Gamma$ is called holonomy and denoted by $\gamma$.

In a Universe with a detectable non-trivial topology [11] each pair of circles is identified with one element of the corresponding holonomy group. An important feature of compact flat manifolds is that any holonomy $\gamma$ of an orientable Euclidean 3-space can always be expressed as a so-called screw motion (in the covering space), consisting of a combination of a rotation (twist) $R(\alpha, \widehat{\mathbf{u}})$ by an angle $\alpha$ around an axis $^{4}$ of rotation $\widehat{\mathbf{u}}$, followed by a translation along a vector $\mathbf{L}=L \widehat{\mathbf{v}}$, say. The action of $\gamma$ on any point $\mathbf{p}$ in the covering manifold is then given by $\mathbf{p} \rightarrow R \mathbf{p}+\mathbf{L}$. When there is no rotational part in the screw motion, i.e., when $\alpha=0$, the holonomy reduces to a pure translation, and its action is exactly the same at every point in space. In this case, the distance between $\mathbf{p}$ and its image by the holonomy $\gamma, \ell_{\gamma}=|\gamma \mathbf{p}-\mathbf{p}|=L$, is the same everywhere. On the other hand, for a general screw motion with $\alpha \neq 0, \ell_{\gamma}$ depends on the location of

to one another. In particular, while the non-flat manifolds are rigid (their compactification lengths being topological invariants in the natural scale provided by the curvature radius), the flat are not, and are therefore free to have different sizes.

4 The choice of axes to describe a screw motion is not unique, but one can always find, and we shall henceforth assume, a rotation axis parallel to the direction of translation. $\mathbf{p}$, and in particular on the distance $r$ between $\mathbf{p}$ and the axis of rotation. Compact flat manifolds are not rigid, in the sense that topologically equivalent flat quotient manifolds, defined by a given holonomy group $\Gamma$, can have different sizes, and therefore their compactification lengths are not fixed. However, since the holonomy group must be a discrete and freely-acting group of isometries of the covering space, the twist parameter $\alpha$ can only assume one of the following discrete values [13 15$]$ :

$$
\alpha=\frac{2 \pi}{n}, \quad \text { with } \quad n=1,2,3,4,6 .
$$

With combinations of these holonomies one can construct the 6 possible classes of flat compact orientable manifolds. In all but one case $\left(E_{6}\right)$, one can obtain the full set of holonomies from combinations of two translational $\left(\alpha=0^{\circ}\right)$ and one screw-motion. Thus, one has the following five manifolds: $E_{1}\left(3\right.$-torus, $\left.\alpha=0^{\circ}\right), E_{2}$ (half turn space, $n=2, \alpha=180^{\circ}$ ), $E_{3}$ (one quarter turn space, $n=4, \alpha=90^{\circ}$ ), $E_{4}$ (one third turn space, $n=3$, $\alpha=120^{\circ}$ ) and $E_{5}$ (one sixth turn space, $n=6, \alpha=60^{\circ}$ ). The sixth manifold, the Hantzsche-Wendt space, has as generators of the holonomy group three screw motions, all with $n=2$ and $\alpha=180^{\circ}$.

The matching circles associated with the holonomy $\gamma=(R(\alpha, \widehat{\mathbf{u}}), \mathbf{L})$ are situated along the intersections of the sphere of last scattering with its images under the holonomies $\gamma$ and $\gamma^{-1}$. Each such pair of circles can be characterized by six angles, three of which are (i) the deviation from antipodicity, $\theta(0 \leq \theta \leq \pi)$, i.e. the complement of the angle between the centers of the matched circles, (ii) the angular radius of the circles, $\nu$ $(0 \leq \nu \leq \pi / 2)$, and (iii) the phase shift $\phi(0 \leq \phi \leq \pi)$, which is the phase angle that measures the shift in the identical distribution of CMB temperature fluctuations of the two circles (see Fig. 1 for details). The remaining three angles give the position of the center of the first circle and the relative orientation of the second circle.

Now, from Fig. 1 1 one can show that for a pair of circles produced by the holonomy $\gamma$, the angular radius $\nu$ is related to the radius of the last scattering surface $\chi_{o b s}$ and the distance $\ell_{\gamma}$ between the observer's position and its nearest image (the length of the shortest closed geodesic) through

$$
\sin \nu=\sqrt{1-\left(\frac{\ell_{\gamma}}{2 \chi_{o b s}}\right)^{2}}
$$

It follows directly from this equation that the only holonomies which generate detectable pairs of circles are those for which $\ell_{\gamma}$ is short enough so that $\ell_{\gamma}<2 \chi_{\text {obs }}$. Furthermore, for these holonomies the shorter the distance $\ell_{\gamma}$ the larger will be the circle radius $\nu$ and thus the holonomy with the shortest distance between the observer and its image, $\ell_{\gamma}$, will produce the matching circles with the largest radii.

The detection of a pair of matching circles would of course imply that the Universe has multiply-connected 


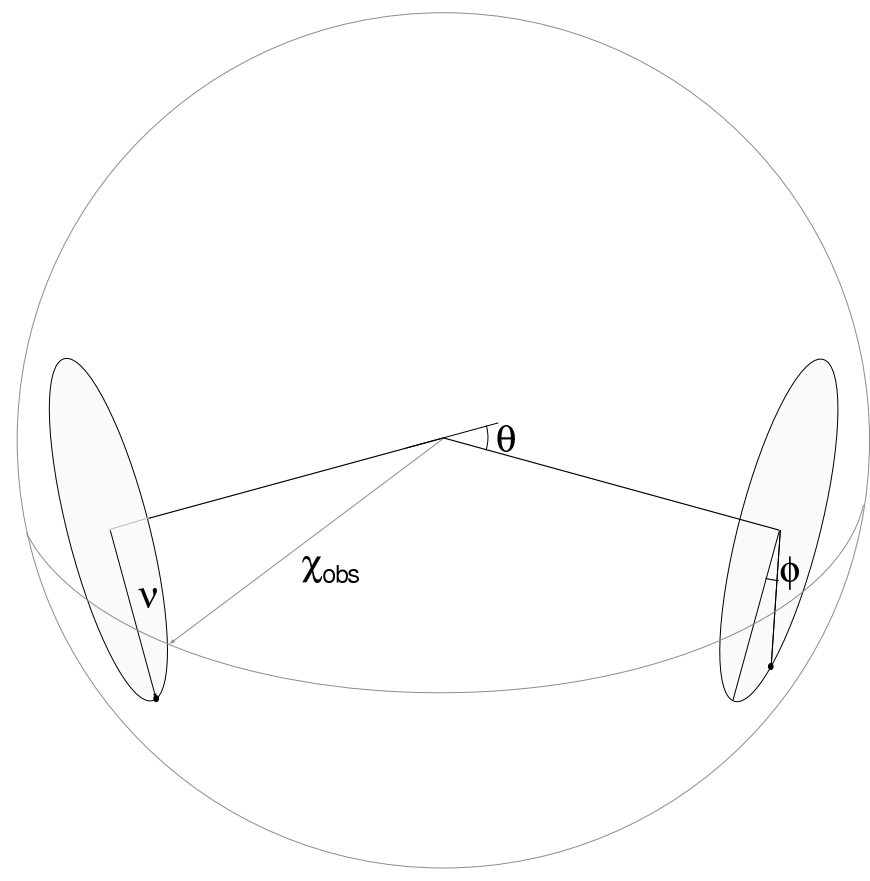

FIG. 1: This figure depicts the circles at the intersection of the last scattering surface with its images. The parameter $\theta$ is a measure of the deviation of the circles from being antipodal, and $\phi$ measures the relative phase between the circles of radius $\nu$.

spatial sections; but beyond that, we want to know how much more information can be gleaned from such a detection. We shall make explicit how and to what extent the detection of a circle-in-the sky could be used to constrain the geometry and topology of the spatial section of the Universe, as well as our position in it. To do so, we must first relate the parameters specifying the circles to the elements of the associated holonomy.

To this end, let us assume that the spatial sections of the Universe have a flat topology so that all holonomies take the form of screw motions. In addition, suppose that we have detected a pair of circles of radius $\nu$ with parameters $\theta, \phi$, which is assumed to be the most readily detectable pair of circles, i.e. the pair associated with the shortest closed geodesic which contains the observer's position or equivalently pairs with the circles with largest radius. ${ }^{5}$

The important question then is, given the parameters $\theta, \phi$ and $\nu$ of a single pair of circles, how to uniquely determine the parameters of the corresponding screw motion,

\footnotetext{
${ }^{5}$ Clearly one might also detect other pairs of circles which are not associated with the first neighboring copies of the CMB sphere. But in such a case these pairs of circles corresponding to neighboring CMB sphere copies, i.e. associated to the shortest closed geodesic, will also be detected, and the pairs could be distinguished by their radii.
}

namely the angle $\alpha$, the compactification length $L$, and the distance $r$ of observer to the axis of rotation?

To answer this question, we need to derive the relation between the parameters specifying the holonomy and those corresponding to the circles. To begin with the screw motion twist angle, $\alpha$ can be uniquely determined by the phase shift $\phi$ and the deviation from antipodicity $\theta$ through the relation

$$
\cos \alpha=\frac{(\cos \phi+1)(\cos \theta+1)}{2}-1
$$

obtained by inverting the expression Eq. (12) of Ref. [12]. Clearly, for a given value of $\alpha$ and $\theta$, there is one and only one possible value of $\phi$. Conversely, the determination of both $\theta$ and $\phi$ specifies $\alpha$. In this way, from Eq. (11) one has that the compact orientable flat manifolds $E_{i}(i=1 \cdots 6)$ define contour curves in the $(\theta-\phi)$ plane, which are the loci of values of the parameters $(\theta, \phi)$ allowed for the circles-in-the-sky of flat universes whose spatial section is one of the associated flat 3-manifolds. The thick lines in Fig. 22 indicate these contour curves (see below for more details about this figure).

One can also show, after some algebra, that the compactification length $L$ and the distance of the observer $r$ to the axis of the screw motion can be written as

$$
L=2 \chi_{o b s} \cos \nu \sqrt{\frac{\cos \theta-\cos \alpha}{1-\cos \alpha}},
$$

and

$$
r=\sqrt{2} \chi_{o b s} \cos \nu \frac{\sqrt{1-\cos \theta}}{1-\cos \alpha} .
$$

respectively. Thus, given the parameters of the circles, $(\theta, \phi, \nu)$, and the radius of the last scattering surface, $\chi_{o b s}$, one can obtain the parameters for the corresponding holonomy $(\alpha, L)$ and the distance $r$ of the observer to the rotation axis (henceforth, the observer's position). Clearly, from Eqs. (5), (4) and (3) one obtains the locus of values of the parameters $(\theta, \phi)$ such that the ratio $r / L$ is constant. This locus defines another family of contour curves in the $(\theta-\phi)$ plane, depicted in Fig. 2 as thin traversing curves for different values of ratio $r / L$. Different combinations of $\theta$ and $\phi$ along each contour correspond to different values of $r$ (in units of the compactification length $L$ ). Figure2 2 shows that for observers situated along the screw motion axis the resulting circles are antipodal $(\theta=0)$, with a relative phase $\phi$ given by the twist angle $\alpha$ [cf. Eq. (3)]. For all observers the deviation from antipodicity $\theta$ becomes larger as the phase $\phi$ decreases. In the limit where the observer is infinitely distant from the axis, $\phi$ becomes zero and $\theta$ becomes equal to $\alpha$ [see Eq. (3)] .

\section{CIRCLES-IN-THE-SKY IN COMPACT ORIENTABLE FLAT UNIVERSES}

Now let us assume observations have detected a single pair of circles of radius $\nu$ with the corresponding parame- 


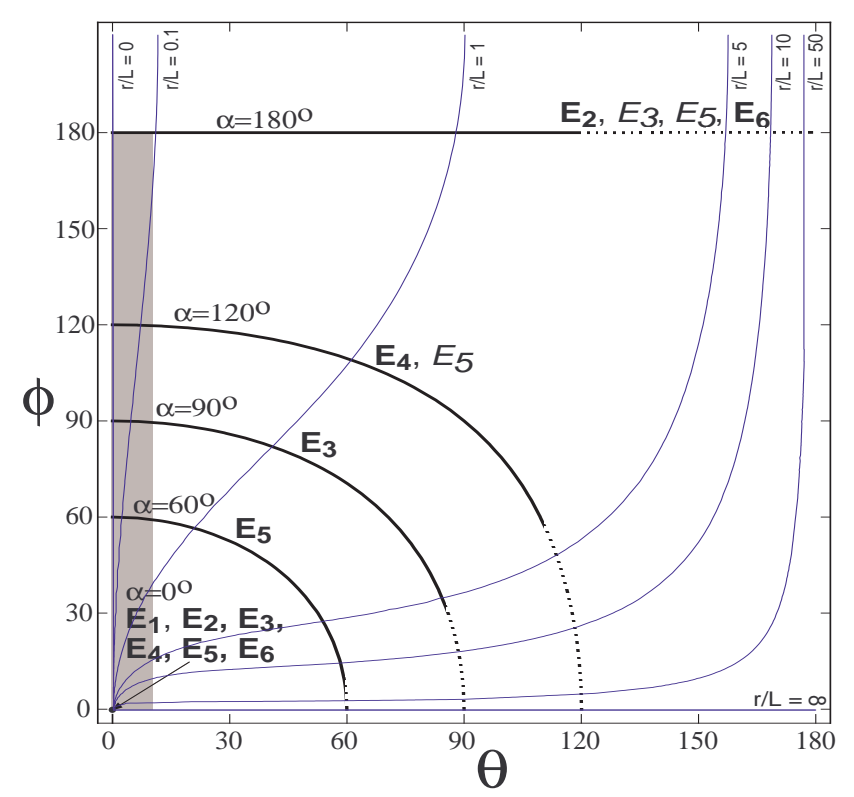

FIG. 2: The allowed combinations of the circles-in-the-sky parameters $\theta$ (the deviation from antipodicity) and $\phi$ (the relative phase between circles) for compact orientable flat manifolds. Each thick contour line corresponds to one allowed class of holonomies, which take the form of a screw motion with twist parameter $\alpha$ given in Eq. (11). The different orientable compact flat manifolds $E_{i}(i=1 \cdots 6)$ that include each holonomy class are indicated; those for which this holonomy may generate the most readily detectable circle pair are in bold. The dotted part in each countour line corresponds to configurations where the circle pairs with the largest radii are always translational, and thus antipodal. In all cases, the compactification length $L$ is not fixed, but the precise combination of $\theta$ and $\phi$ will depend on the position of the observer, as depicted in the light contours corresponding to different distances $r$ from the axis of the screw motion (in units of $L$ ). The shaded area corresponds to the parameter values that have been probed in the recent searches for antipodal and nearly antipodal circles in CMB maps [6, 7].

ters $(\theta, \phi)$. As we mentioned above, in compact orientable flat manifolds the screw motion angle $\alpha$ is restricted to the values given by Eq. (11). Also, for each of the allowed values, Eq. (3) becomes a finite set of one-to-one relations between $\theta$ and $\phi$, as is shown in Fig. 2. Thus, the detection of a pair of circles will allow the knowledge of the parameters $\theta$ and $\phi$ to readily determine whether or not the geometry of the spatial section of the Universe is Euclidean (flat), by checking whether the observed values of $\theta$ and $\phi$ lie on one of the contours (thick curves) shown in Fig. 2. If they do not (after taking into account observational uncertainties) then this is a clear indication that the geometry is non-Euclidean. Conversely, if the parameters $\theta$ and $\phi$ of the detected pair lie, within observational uncertainty limits, on one of these thick contours curves, we would conclude that the underlying spatial geometry is most likely Euclidean (flat). ${ }^{6}$

Now if it is found that the geometry is indeed Euclidean, we then wish to establish to what extent the topology of the spatial section of the Universe can be determined, given such a detected pair of circles. The list of possibilities of compact orientable flat manifolds is summarized in Table 【. We indicate in each case the maximum deviations from antipodicity of the circles-in-thesky for which a non-translational holonomy may generate the most readily detectable pair of circles (see Ref. [8] for details).

\begin{tabular}{cccc}
\hline \hline Symbol & Manifold & $n$ & $\theta_{\max }$ \\
\hline$E_{1}$ & three-torus & $1,1,1$ & $0^{\circ}$ \\
$E_{2}$ & half turn space & $1,1,2$ & $120^{\circ}$ \\
$E_{3}$ & quarter turn space & $1,1,4$ & $86^{\circ}$ \\
$E_{4}$ & third turn space & $1,1,3$ & $109^{\circ}$ \\
$E_{5}$ & sixth turn space & $1,1,6$ & $59^{\circ}$ \\
$E_{6}$ & Hantzsche-Wendt space & $2,2,2$ & $120^{\circ}$ \\
\hline \hline
\end{tabular}

TABLE I: Multiply-connected flat orientable manifolds and the maximum deviation from antipodicity of the circles-inthe-sky for each manifold for which a non-translational holonomy may generate the most readily detectable pair of circles(i.e., with the largest radii). In all cases the screw motion twist parameters can only take certain values of the form $\alpha=2 \pi / n$; the values of $n$ for the holonomy group generators are also indicated. Note that $n=1$ corresponds to a translation.

Table $\square$ and Fig. 2 show that each of the 6 possible classes of holonomies corresponds to the different values of $\alpha$ [cf. Eq. (1)], which in turn belong to the holonomy group of one or more of the compact orientable flat manifolds.

At first sight, this seems to indicate one-to-many correspondence between the values of the twist angle $\alpha$ [obtained from the detected angular parameters of circles $(\theta, \phi)]$ and the list of flat orientable compact manifolds $E_{i}(i=1 \ldots 6)$ with their associated holonomies.

However, as our previous work [8] details, some elements of a holonomy group can never produce the pair of circles with the shortest distance between the observer and its image, no matter where in the manifold the observer happens to be. For instance, although a screw motion with a twist of $120^{\circ}$ exists in the holonomy group of $E_{5}$, being the square of its non-translational generator with a twist of $60^{\circ}$, it can be shown that the distance between any point and its image by the former holonomy is always larger than the corresponding distance by the

\footnotetext{
${ }^{6}$ The values along the contours are in fact compatible with some positions of the observer for certain curved manifolds, but the full set of possible combination of the latter densely span the $\theta-$ $\phi$ plane, and thus the set of values corresponding exactly to the contour curves correspond to a zero-measure set of observers for each potential non-Euclidean manifold.
} 
latter. Thus, if a pair of circles associated with a twist of $120^{\circ}$ is detected, it means that either the spatial section of the Universe is $E_{4}$, or it is $E_{5}$ but there is another pair of matching circles, of larger radii (and thus more readily detectable), the position of which can be worked out from the position of the centers of the circles and from Eqs. (3), (4) and (5). ${ }^{7}$ If, on the other hand, no other pair is detected in a full-sky search, then merely the presence of this pair of circles is sufficient to ensure that the spatial section has an $E_{4}$ topology. In this way, a non-antipodal circle pair detection with parameters on any contour line in Fig. 2, is compatible with only one flat compact orientable manifold, apart from one case: a circle pair with phase shift of $\phi=180^{\circ}$ for any value of $\theta$ (implying a screw motion with a twist of $\alpha=180^{\circ}$ ) may be the most readily detectable circle pair in either $E_{2}$ and $E_{6}$ (and can also be present in $E_{3}$ and $E_{5}$, albeit necessarily alongside some other circle pair with larger radii). Thus, the observation of a single pair of nonantipodal circles removes the ambiguities in determining the spatial topology and, except for the degeneracy between $E_{2}$ and $E_{6}$, it allows one to fully reconstruct the entire holonomy group (apart from at most two compactification lengths associated with the group generators not directly observed), as well as to determine observer's position within the compact manifold.

This leaves out the antipodal case given by $\theta=0=\phi$, where the circles are back-to-back with no phase difference. As can be seen from Fig. 2, this is a totally degenerate case, since for any flat 3-manifold a suitable choice of position and compactification lengths guarantee that a translation generates the only detectable circle pair. Note, however, that this case has been in principle ruled out by the searches that have already taken place.

In closing, we note that our results can also be useful to devise search strategies for circles-in-the-sky in CMB maps that reduce the size of the parameter space that needs to be numerically searched. This is potentially significant because a pair of matching circles in the celestial sphere is defined by six angular parameters. A six-parameter search in high-resolution maps to be produced from the ongoing Planck mission [9] would be prohibitively time-consuming. By combining our previous result according to which the deviation from antipodicity is very likely to be small for circle pairs in very nearly flat universes, with the connection shown here to exist between two of the circle pair parameters in flat universes, our results suggest that future searches should be confined to combinations of $\theta$ and $\phi$ given by Eq. (3) restricted to Eq. (1). Clearly, in order to carry out a new search for nearly back-to-back pairs of circles, an additional full range of $\phi$ values for small values of $\theta$ should be undertaken. In this way, one would effectively reduce

\footnotetext{
7 Clearly, we are ignoring the possibility of having such circles hidden by galactic contamination.
}

the dimension of the survey parameter space by one, ensuring a significant reduction in computational time.

\section{CONCLUDING REMARKS}

The existence of pairs of correlated circles in CMB maps with the same distribution of temperature fluctuations - the so-called circles-in-the-sky - is a generic prediction in a Universe with a detectable nontrivial cosmic topology, regardless of the background geometry. Detecting such circle pairs would provide a measure of the value of their corresponding parameters $(\theta, \phi, \nu))$.

We have made a detailed study of the extent to which the detection of a single pair of circles in CMB maps can be used to determine the geometry and topology of the spatial sections of the Universe.

We have shown that as long as we detect the pair of circles which is the most readily detectable one, i.e. associated with the nearest (topological) copies of the last scattering surface, then the analysis of the corresponding circle parameters given in this paper is sufficient to determine whether the geometry is Euclidean (flat) or curved, within the observational uncertainties. In the former case, we have also shown that we can fully determine the topology of the spatial sections of the Universe, apart from one case.

Given the upcoming high resolution data from Planck [9], it is conceivable that pairs of circles-in-thesky can be detected by more comprehensive searches than those so far undertaken [6, 7]. However, any detection is likely to be partial, in the sense that it is very unlikely that one would have access to a complete set of generators of the holonomy group from the observable circles-in-thesky. In spite of this, what we have shown in this paper is that even the most partial detection, i.e. the observation of a single pair of circles, is in principle sufficient to determine whether the spatial section of the Universe is flat or curved. Furthermore, if it is flat, we have shown how one can determine the topology of the spatial section of the Universe from this minimal detection.

Finally our results have an important consequence for the future search for circles-in-the-sky strategies. We had previously shown that searches so far undertaken (if confirmed) would be sufficient to in principle exclude the possibility of a very nearly flat universe with a non-trivial topology [4, 5]. In the light of this result, our results in the present paper suggest where in the parameter space the searches for circles-in-the-sky one should concentrate on, thus significantly reducing their computational costs.

\section{Acknowledgments}

M.J. Rebouças acknowledges the support of FAPERJ under a CNE E-26/101.556/2010 grant. This work was also supported by Conselho Nacional de Desenvolvimento Científico e Tecnológico (CNPq) - Brasil, under grant No. 
475262/2010-7. M.J. Rebouças and R. Tavakol thank, respectively, $\mathrm{CNPq}$ and $\mathrm{PCI}-\mathrm{CBPF} / \mathrm{MCT} / \mathrm{CNPq}$ for the grants under which this work was carried out.
[1] G. F. R. Ellis, Gen. Rel. Grav. 2, 7 (1971); M. LachièzeRey and J.P. Luminet, Phys. Rep. 254, 135 (1995); G.D. Starkman, Class. Quantum Grav. 15, 2529 (1998); J. Levin, Phys. Rep. 365, 251 (2002); M.J. Rebouças and G.I. Gomero, Braz. J. Phys. 34, 1358 (2004); M.J. Rebouças, A Brief Introduction to Cosmic Topology, in Proc. XIth Brazilian School of Cosmology and Gravitation, eds. M. Novello and S.E. Perez Bergliaffa AIP Conference Proceedings vol. 782, p 188. Also arXiv:astro-ph/0504365

[2] N.J. Cornish, D. Spergel, and G. Starkman, Class. Quantum Grav. 15, 2657 (1998)

[3] H. Fujii and Y. Yoshii, Astron. Astrophys. 531, A171 (2011); H. Fujii and Y. Yoshii, arXiv:1103.1466 [astro-ph.CO]; G. Aslanyan and A.V. Manohar, arXiv:1104.0015 [astro-ph.CO]; M. Marcolli, E. Pierpaoli, and K. Teh, Commun. Math. Phys. 304, 125 (2011); R. Aurich and S. Lustig, Class. Quant. Grav. 28085017 (2011); M. Marcolli, E. Pierpaoli, K. Teh, arXiv:1012.0780 [hep-th]; B. Mota, M.J. Rebouças, and R. Tavakol, arXiv:1007.3466 [astro-ph.CO]; S.D.P. Vitenti, M.P. Lima, and M.J. Rebouças, arXiv:1008.0832 [astro-ph.CO]; h Published in Astronomy and Relativistic Astrophysics: New Phenomena and New States of Matter in the Universe. Eds. C.A.Z. asconcellos, B.E.J. Bodmann, H.Stoecker, M.J. Rebouças, V. B. Bezerra, W. Greiner. World Scientific, 2010, p219; P. Bielewicz and A.J. Banday, arXiv:1012.3549 [astro-ph.CO]; i B.F. Roukema, arXiv:1002.3528 [astro-ph.CO]; R. Aurich, H.S. Janzer, S. Lustig, and F. Steiner, Class. Quantum Grav. 25, 125006 (2008); R. Aurich, Class. Quant. Grav. 25225017 (2008); M. Kunz, N. Aghanim, A. Riazuelo, and O. Forni, Phys. Rev. D 77, 023525 (2008); M. Kunz, N. Aghanim, L. Cayon, O. Forni, A. Riazuelo, and J.P. Uzan, Phys. Rev. D 73, 023511 (2006); R. Aurich, S. Lustig, and F. Steiner, Class. Quantum Grav. 22, 2061 (2005); R. Aurich, S. Lustig, and F. Steiner, Class. Quantum Grav. 22, 3443 (2005); B.F. Roukema, B. Lew, M. Cechowska, A. Marecki, and S. Bajtlik, Astron. Astrophys. 423, 821 (2004); A. Linde, JCAP 0410, 004
(2004); G.I. Gomero, A.F.F. Teixeira, M.J. Rebouças, and A. Bernui, Int. J. Mod. Phys. D 11, 869 (2002); G.I. Gomero, M.J. Rebouças, and A.F.F. Teixeira, Phys. Lett. A 275, 355 (2000). G.I. Gomero, M.J. Rebouças, and R. Tavakol, Class. Quantum Grav.18, L145 (2001); R. Aurich, S. Lustig, F. Steiner, Phys. Rev. Lett. 94. 021301 (2005); R. Aurich, S. Lustig, F. Steiner, H. Then, Class. Quant. Grav. 21, 4901 (2004).

[4] B. Mota, G.I. Gomero, M.J. Rebouças, and R. Tavakol, Class. Quantum Grav. 21, 3361 (2004); B. Mota, M.J. Rebouças, and R. Tavakol, Int. J. Mod. Phys. A 20, 2415 (2005).

[5] B. Mota, M.J. Rebouças, and R. Tavakol, Phys. Rev. D. 78, 083521 (2008).

[6] N.J. Cornish, D.N. Spergel, G.D. Starkman, and E. Komatsu, Phys. Rev. Lett. 92, 201302 (2004).

[7] J.S. Key, N.J. Cornish, D.N. Spergel, and G.D. Starkman, Phys. Rev. D 75, 084034 (2007).

[8] B. Mota, M.J. Rebouças and R. Tavakol, Phys. Rev. D. 81, 103516 (2010).

[9] "The Scientific Programme of Planck", The Planck Collaboration, ESA-SCI(2005)1, arXiv:astro-ph/0604069.

[10] Thurston, W.P., Three-Dimensional Geometry and Topology. Vol.1, Edited by Silvio Levy, Princeton University Press (1997).

[11] G.I. Gomero, M.J. Rebouças, and R. Tavakol, Class. Quantum Grav. 18, 4461 (2001); G.I. Gomero, M.J. Rebouças, and R. Tavakol, Int. J. Mod. Phys. A 17, 4261 (2002); J. Weeks, Mod. Phys. Lett. A 18, 2099 (2003); B. Mota, M.J. Rebouças, and R. Tavakol, Class. Quantum Grav. 20, 4837 (2003).

[12] G.I. Gomero, arXiv:astro-ph/0310749

[13] C. Adams and J. Shapiro, American Scientist 89, 443 (2001).

[14] B. Cipra, Whats Happening in the Mathematical Sciences, American Mathematical Society, Providence, RI, 2002.

[15] A. Riazuelo, J. Weeks, J.P. Uzan, R. Lehoucq, and J.P. Luminet, Phys. Rev. D 69, 103518 (2004). 